\title{
Improving Learning Outcomes by Designing Engaging Educational Tools
}

\author{
John Casey, Nilufar Baghaei \\ Unitec Institute of Technology \\ Private Bag 92025, Victoria Street West \\ Auckland 1142, New Zealand \\ \{jcasey,nbaghaei\}@unitec.ac.nz
}

\author{
Kalpana Nand \\ Mission Heights Primary School \\ 103 Jeffs Road, Flat Bush \\ Manukau 2016, New Zealand \\ knand@mhp.school.nz
}

\begin{abstract}
The success rate of computer games in engaging children has prompted educational researchers to investigate if similar techniques can be used to engage children with learning. In this paper, we present the results of a study conducted with 120 primary school children, in which two versions of our proposed educational tool (Features Enriched Game (FEG) vs Feature Devoid Game (FDG)) were used for four weeks to teach primary school curriculum areas of Numeracy and Te Reo Maori language. The effectiveness of the educational tool was measured using a pre-test and a post-test, as well as the frequency and duration of time on playing the game. The results showed that the FEG version enhanced children's learning - it was more effective as an educational tool in both Numeracy and Te Reo curriculum areas, when compared to the FDG version. In the case of Numeracy, the increase in scores was twice as much as the FDG version and in the case of Te Reo (Maori Language) it was five times as much. Similar results were also shown by other indicators such as time and frequency. Finally, the results showed that the FEG version of the game was more popular with children.
\end{abstract}

Keywords-Designing Educational Tools; Learning; Games; Engagement; Children

\section{INTRODUCTION}

The use of computer games as common vehicles for education, as opposed to pure entertainment, has gained immense popularity in recent years. Several studies such as [1] have investigated the negative effects of computer games on children as well as generally on society, especially the effects of the violent themes contained in a large proportion of games. Some of these studies have also looked at the effect of extended periods of game playing on children.

There are a wide range of computer games available today. A lot of attention has recently been diverted towards the effect of playing games on children's learning (e.g. [2] \& [3]). A key factor is the capacity of such games to engage the players for extended periods of time. There are certain attributes of computer games which contribute to how well they are received by the players. Designers of educational tools can integrate these attributes to maximise the tools' effectiveness in increasing learning outcomes, level of engagement and motivation. According to a study conducted by Prensky [4], a prerequisite of successful learning is motivation. He argues that a lot of what is in the curriculum is not motivating for students. Yet the same children are motivated and excited to play video games for long duration. One way of getting children motivated is to design educational tools with the aim of making them as engaging and motivating as popular commercial games. These tools can be integrated with the curriculum to enhance children's learning.

In our earlier work, we investigated the main characteristics of engaging computer games (briefly described in Section 3) and designed an educational tool with those characteristics embedded into it [5]. This paper seeks to examine the effectiveness of our proposed educational tool in enhancing students' knowledge of Numeracy and Maori Language - two subjects heavily used in New Zealand primary school curriculum

The remainder of this paper is organised as follows. Section 2 reports on the current literature. Section 3 briefly describes our proposed educational tool. We then present the research questions followed by the evaluation study and the results in Section 4. Conclusions and future work are discussed in Section 5.

\section{RELATED WORK}

The use of technology, such as computer games, to enhance student achievement in the classroom is a timely topic that permeates a lot of educational literature today. Video and computer game design have been studied by various researchers interested in finding out how different aspects of the game design could be utilised in developing educational games (e.g. [6]; [7]). The increase in the popularity of computer games and recent developments in information and computer technologies have attracted researchers to investigate the learning benefits of computer games. Studies have shown that game playing makes up a vital element of a child's cognitive and social development e.g. [8]. They argue that children learn more from playing and carrying out "handson" activities than by being simply asked to "recite" information from books. Children learn by playing with others, creating and improving their zone of proximal development; as they play, they are more involved in carrying out complex activities [9]. The learning aspect of computer 
games has been further endorsed by [2]. In this study, the researchers proposed a set of design guidelines that can be ideally applied to any game to teach children how to manage their diabetes. The preliminary results of their research showed that users enjoyed playing the game and they believed their knowledge of diabetes increased as a result of playing the game. Other examples of educational and health-related games include [10]. A lot of games stimulate curiosity and the outcome, i.e., the desire to win is what attracts players to playing any game. For any game to be successful, it must be able to engage the player.

Based on our experience, most of the educational games available in New Zealand primary schools are not motivating enough for students. These games lack the fun factor. Children are not as motivated to play these games as they are to play popular computer and video games at home. Most of the games that do exist are usually the basic grill and drill practice models. There is a need to develop useful and instructional computer games, which are relevant to the current New Zealand curriculum and can be integrated in the day to day learning. In our previous study [5], we investigated the main characteristics of computer games used to engage player for a long period of time. We then designed an educational tool based on the feedback we collected.

\section{GAME DESIGN}

We selected a group of 120 children aged between $9-10$ at Glen Eden Primary School in Auckland, NZ. They were given a questionnaire and were asked to choose 3 features (from a given list) of computer games that they found most appealing. The following game attributes were most appealing [5]:

- Challenges (CH): having different levels in the game

- Feedback (FB): knowing how many points were scored

- Graphics (GH): having realistic graphics

The participants were also asked to select the curriculum area in which they preferred the tool to be designed in. The Topics Related part included Science, Social Studies, Technology and Te Reo (Mario language). A vast number of children were interested in playing numeracy games. Some of the reasons given as to why they wanted a numeracy game developed included: "I want to get better at math", "I want to learn my multiplication facts", "Learning math in a game will be a fun way to learn" and "I don't like math so playing a game and learning will be better".

Driven by the three main characteristics identified by the participants (i.e. $\mathrm{CH}, \mathrm{FB}$ and $\mathrm{GH}$ ), a variety of open source games were examined. We felt that the Java-based open source game "Who wants to be a Millionaire"(http://quizshow.sourceforge.net/download.html) is a suitable option to choose for the preliminary evaluation (see Fig. 1). It was also identified as one of the games children enjoyed playing; hence modifications were made to incorporate engaging features into it.
The game is based on a television game show in which the participants are offered cash prizes for correctly answering a series of multiple-choice questions in the order of increasing difficulty levels. This game can be configured easily to include any content. New content can be added by including the questions at various levels as a text file. Choosing an incorrect answer at any point in the game ends the current session, with a feedback message saying the game can be played again from the beginning. Depending on when the incorrect answer is given, the player can leave with either no money or a certain amount. The amount a player can leave with depends on the level reached.

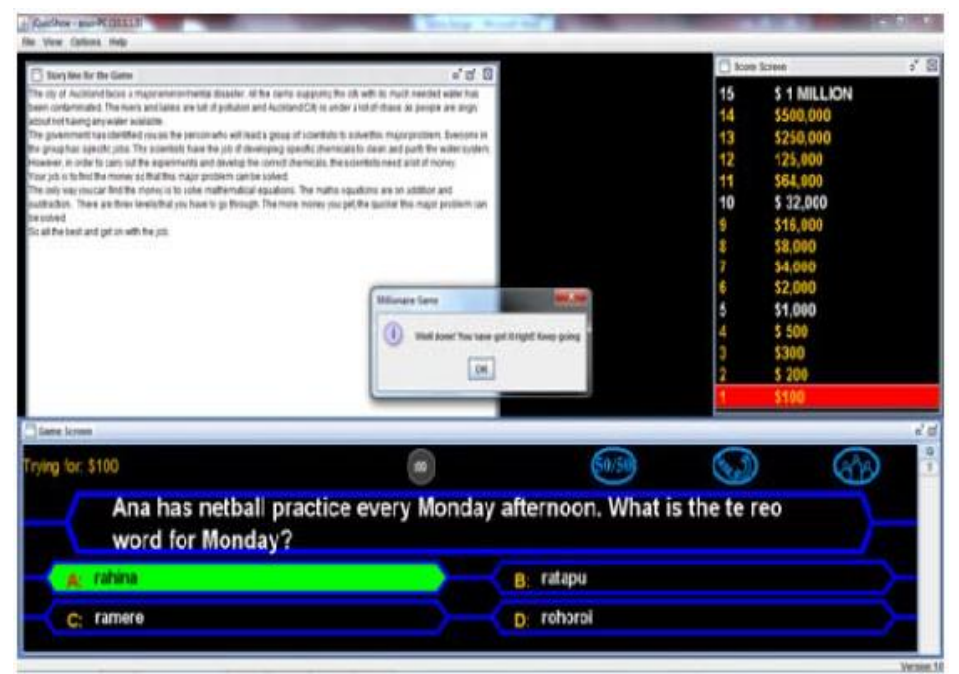

Fig. 1. Screenshots from the game "who wants to be a millionaire?"

The game designed for this study had three levels indicated by an amount written in white font compared to the rest of the amounts which are written in yellow font (see Fig. 1). Once a player passes a level1 indicated by the amounts $\$ 1,000, \$ 32,000$ and $\$ 1$ million, the player can leave anytime with the money associated with the highest previous level reached. This applies in both cases: when a player voluntarily chooses to leave the game and/or when the player gets an incorrect answer.

The two subject areas chosen for this study were Numeracy and Te Reo (Maori language). Numeracy was chosen because a large number of students had identified numeracy in the questionnaire as one of the areas they wanted to play games in. The New Zealand Numeracy Curriculum was used in order to determine the level of question suitable for the children selected for the study. In order for the game to be enjoyable and engaging, it was necessary that the players were given the type of questions of which they had prior knowledge and which were not extremely difficult or "boringly" easy (e.g. a good solution was to provide a progressive level of skills). Their teachers were consulted and the numeracy levels of the children were taken into consideration. Te Reo (Maori Language) was chosen to be the second subject to teach, since this was an area of learning in which there were very limited educational games. Te Reo is a component of the NZ Primary School curriculum. The 
teachers of the selected group of students were again consulted to find out what competency level the students were in Te Reo. The questions designed for the Te Reo game were then compiled in line with the children's competence. The questions were based around identifying everyday items, recalling numbers, naming the colour range, as well as naming the days of the week. The children in the study group were expected to have prior knowledge on these topics.

We developed two versions. The first version was a Feature Enriched Game (FEG) which had extensive use of the three identified features (i.e. $\mathrm{CH}, \mathrm{FB} \& \mathrm{GH}$ ) and the second version, a Feature Devoid Game (FDG) had overt absence of these features. To find out more about the proposed features and how they were integrated into the FEG version of the game, please refer to our earlier work [5].

\section{RESEARCH QUESTIONS \& EVALUATION STUDY}

Our research questions are as follows: 1) Will adding $\mathrm{CH}, \mathrm{FB}$ and $\mathrm{GH}$ characteristics to the game (i.e. FEG version) enhance children's learning? 2) Does using different subjects (Numeracy vs Te Reo) result in different learning outcomes? 3) Do children enjoy using the proposed educational tool with those characteristics embedded?

The study was conducted with 120 children aged 9-10 at Glen Eden Primary School in Auckland. The participants were randomly divided into a Control group and a Test group of 60 students each. Both groups were pre-tested firstly on the numeracy learning outcomes. The Test group was given the FEG version to play over a period of two weeks and the Control group was given the FDG version to play over two weeks. Both groups were given post tests on the numeracy learning outcome. Both groups were then pre-tested on the Te Reo learning outcome. The test group was given the Te Reo FEG version to play over a period of two weeks and the Control group was given the Te Reo FDG version to play over two weeks. Both groups were given post tests on the Te Reo learning outcome.

Both FEG and FDG versions of the game were installed on the 12 available computers in the school library and as time permitted, pupils in groups of 12 were given the games to play in a separate room with the computers. Both Control and Test groups played at different times and were not able to see what version of game each group was playing. There was a deliberate attempt to keep the two group's playing times separate. The students were allowed to play the game for about 20 minutes without any interference from the researcher or any of the other teachers.

Measuring children's learning was our main dependent variable. In order to do that, we used a pre-test, post-test and interaction logs. The pre-test was conducted to measure student knowledge before using the educational tool and the post-test was used to measure the learning outcome after using the educational tool. The questions in the tests were similar to the ones used by teachers in assessing their students in numeracy and their knowledge of Maori language. The pretest and the post-test for each of the curriculum areas were done using the same questions. This gave us a direct measurement of the change in the learning outcome. The results are reported in Table $1 \mathrm{a} \& 1 \mathrm{~b}$.

TABLE Ia. Statistics for the Pre and Post Scores for the Numeracy experiment

\begin{tabular}{|l|c|c|c|c|}
\hline & \multicolumn{2}{|c|}{ Control Group } & \multicolumn{2}{c|}{ Test Group } \\
Statistic & Pre Test & Post Test & Pre Test & Post Test \\
\hline \hline Count & 60 & 60 & 60 & 60 \\
Average & 12.12 & 12.97 & 12.87 & 14.77 \\
Std. Dev. & 4.30 & 4.21 & 4.55 & 3.51 \\
Relative Std. Dev. (\%) & 35.5 & 32.5 & 35.4 & 23.8 \\
\hline
\end{tabular}

TABLE Ib. Statistics for the Pre and Post Scores for the Te Reo experiment

\begin{tabular}{|l|c|c|c|c|}
\hline & \multicolumn{2}{|c|}{ Control Group } & \multicolumn{2}{c|}{ Test Group } \\
Statistic & Pre Test & Post Test & Pre Test & Post Test \\
\hline \hline Count & 60 & 60 & 60 & 60 \\
Average & 8.35 & 8.85 & 10.52 & 14.18 \\
Std. Dev. & 3.47 & 3.41 & 3.22 & 3.42 \\
Relative Std. Dev. (\%) & 41.6 & 38.6 & 30.7 & 24.1 \\
\hline
\end{tabular}

As we can see, the average scores for both domains have increased after playing both versions of the game. For numeracy domain (Table Ia), the average for the control group has increased from 12.12 to 12.97 and for the test group, has gone up from 12.87 to 14.77 . In addition, the absolute score for these equate to an increase of 0.85 or $7 \%$ for the control group and an increase of 1.9 or $14.8 \%$ for the test group. Thus, the percentage increase in the mean score is twice as much for the FEG compared to the FDG game. Comparison of the posttest scores for the control and the test groups (12.97 compared to 14.77) also shows that the FEG was more effective in raising the performance level of the students. The T-Test values are $3.63 \times 10^{-10}$ for the Control group and $1.31 \times 10^{-31}$ for the Test group. Both values are orders of magnitude smaller than 0.05 , showing that the change in the learning outcome (post-test vs pre-test) was statistically significant for both groups. Additionally, the T-Test value for the Test group is orders of magnitude smaller than the Control group T-Test value, implying a significant effect of the FEG.

The corresponding figures for the Te Reo game is 0.5 or $6 \%$ increase for the control group compared to 3.66 or $34.8 \%$ for the test group. This gives us an approximately 6 fold increase in the learning score for the Te Reo experiment. The results in the case of the Te Reo curriculum also show a similar pattern to the Numeracy curriculum, in that the test group performed better compared to the control group.

Table II shows the average values of some of the other attributes of the experiments that were extracted from the log files. The participants in the Test group attempted more questions in average, provided more correct answers, spent more time playing the game and reached more levels compared with the Control group - this indicates that the FEG version was better utilised compared with the FDG.

Another fact apparent from Table II is that the values of the attributes are slightly higher for the Te Reo test group compared to the Numeracy test group. This affirms the results 
from the learning outcome scores; that the game was more effective for the Te Reo curriculum compared to Numeracy.

TABLE II. Interaction logs for Test and Control Groups for both domains

\begin{tabular}{|l|c|c|c|c|c|c|}
\hline & \multicolumn{2}{|c|}{ Numeracy } & \multicolumn{2}{c|}{ Te Reo } & \multicolumn{2}{c|}{ Numeracy plus Te Reo } \\
Av. per participant & Control & Test & Control & Test & Control & Test \\
\hline \hline No. of participants & 60 & 60 & 60 & 60 & 60 & 60 \\
No. of questions attempted & 9 & 11.5 & 8 & 13.5 & 8.5 & 12.5 \\
No of conrect responses & 8 & 10.5 & 7 & 12.5 & 7.5 & 11.3 \\
Time spent playing game (mins) & 9.19 & 10.44 & 9.21 & 10.5 & 9.2 & 10.5 \\
Level reached & 1.8 & 2.3 & 1.6 & 2.63 & 1.7 & 2.5 \\
\hline
\end{tabular}

After playing the FEG and the FDG versions of the game over a period of four weeks, the students were given a questionnaire to fill out, in order to collect information about their perception of the game. The test group indicated that they enjoyed playing game more than the participants in the control group. The results also showed that the sound effects, the visual effects, the level of challenges in the game and the feedback messages contributed more to their engagement. The test group is also more likely to want to play the game again.

\section{CONCLUSIONS \& FUTURE WORK}

In this paper, we examined the effectiveness of our proposed educational tool in enhancing students' knowledge of Numeracy and Maori Language - two subjects heavily used in New Zealand primary school curriculum. The tool is designed based on the characteristics of engaging computer games identified in our previous study, i.e. graphics $(\mathrm{GH})$, feedback (FB) and challenge $(\mathrm{CH})$.

We conducted the evaluation study with 120 primary school children, at Glen Eden Primary School in Auckland over a period of four weeks. The main dependent variable used was the amount of learning that took place, measured with the use of pre-test and post-test and user interaction data. The results showed that the proposed features embedded into the learning tool were effective in significantly improving learning outcomes. The results also showed that the test group did significantly better when working on the Te Reo questions than Numeracy. The much greater increase in the learning outcome score for Te Reo indicates that the educational tool was especially effective for this curricular.

The difference in results can be attributed to the difference in the types of learning involved in the two curricula. Te Reo involves fact-based learning where the student is required to learn and recall facts. Factual learning focuses on the attainment and storage of information to be retrievable on demand [11]. The type of questions asked in the Te Reo version was based on having a prior knowledge about the topic. Hence the children were asked to recall factual information that they had prior knowledge about. They were able retain this information after playing the game for a period of four weeks. On the other hand, the Numeracy learning task is based on problem solving and exploring; it involves more fact manipulation operations and various intermediate steps in order to arrive at the final answer. The intermediate steps were not fully supported in the current version of the tool designed for this study. This game reinforces previously introduced skills and concepts. It doesn't teach students new concepts. Constructivist theorists (e.g. [12]) assert that when students construct personal knowledge derived from meaningful experiences, they are much more likely to retain and use what they have learned. Hence any learning tool, such as the game designed for this study, should be able to suitably support this.

Using games to teach the curricula helps in motivating the students as well as keeping them engaged through the duration of the learning task. Our results show that enriching the educational tool with certain features goes even further in improving the learning outcomes. Although the study was conducted with primary school children, our methodology can be applied to subjects related to Science and Engineering.

An immediate future work identified is to adapt the game for more cognitive based learning tasks. It would be interesting to see if the effectiveness of the FEG version would also be valid for other curriculum areas. We also plan to conduct a long-term study to find out if there will be significant increase in learning outcomes and amount of enjoyment as opposed to a two week study. We believe our research paves the way for the systematic design and development of full-fledged engaging educational tools.

\section{REFERENCES}

[3] Fisch, S. M. (2005). "Making educational computer games "educational". In Proc. Int. Conference on Interaction design and children, Boulder, CO.

[4] Prensky, M. (2001). "Digital Game-Based Learning". McGraw Hill: New York.

[5] Nand, K., Baghaei, N., Casey, J. "Play \& Learn: Designing Engaging Educational Games for Children". Cognitive Technology Journal, vol. 18, no. 1, 2013.

[6] Dondlinger, M. J. (2007). "Educational Video Game Design: A Review of the Literature". Journal of Applied Educational Technology, 4(1), 21-31.

[7] Malone, T. W. (1981). "Toward a theory of intrinsically motivating instruction". Cognitive Science, (4), 333-369.

[8] Csikszentmihalyi, M. (1990). "Flow: The psychology of optimal experience". London: Harper Perennial.

[9] Vygotsky, L. (1976). "Play: Its role in development and evolution, chapter Play and its role in the mental development of the child", pages 537-554. New York, NY: Penguin Books.

[10] Alankus, G., Lazar, A., May, M. and Kelleher, C. "Towards Customizable Games for Stroke Rehabilitation". In Proc. Int Conference on Human Factors in Computing Systems (CHI 2010), ACM Press: Atlanta, GA, USA.

[11] Sutherland, R. D. (1969). "Some thoughts on conceptual learning and information retrieval". broadside distributed at Illinois State University (March, 1969).

[12] Piaget, J. (1970). "The Science of Education and the Psychology of the Child". New York, NY: Grossman 